\title{
Mass Spectroscopic Analysis of Hair from Archaeological Remains
}

\author{
Ivan Kempson*, William Skinner*, Paul Kirkbride**, Andrew Nelson***, Ron Martin***. \\ * Ian Wark Research Institute, University of South Australia, Mawson Lakes, 5095, AUSTRALIA \\ ** Forensic Science South Australia, 21 Divett Place, Adelaide 5000, AUSTRALIA \\ *** Dept. of Chemistry, University of Western Ontario, London, Ontario, N6A 5B7, CANADA.
}

Time-of-Flight Secondary Ion Mass Spectrometry (ToF-SIMS) and Inductively Coupled Plasma Mass Spectrometry (ICP/MS) have been used to analyse elemental and molecular species in hairs from a variety of individuals of archaeologically significance. Results from approximately 1000 to 1500 year old mummified remains excavated from Pacatnamu, Peru, and a 550 year old Ice-man discovered in British Colombia, Canada, named Kwaday Dan T'sinchi (KDT) are discussed. A technique was developed for the longitudinal sectioning of hair samples (Figure 1) for analysis by ToF-SIMS to compare the internal and external concentrations and were compared with results from ICP/MS in an effort to identify biogenic uptake and diagenetic contamination.

The nutritional source of growing hair is the blood supply, which contains traces of anything consumed by the individual. These xenobiotics and their metabolites become incorporated into the growing hair matrix. Hair analysis has been utilised in the study of nutrition, dietary habits, exposure to toxins, and drug abuse. However controversy exists, particularly in trace element research, regarding the role of exogenous contaminants. A full understanding of the stability of the hair matrix over time and hence its value in the study of ancient cultures is yet to be realised.

The ToF-SIMS method implemented here is not a bulk method, as is typically used and hence, has an ability to distinguish contamination from endogenous contributions. This method is valuable for the study of rare, valuable and/or minute samples, such as those from mummies where it is beneficial to use as little material as possible. Research performed by the authors has aimed at increasing the level of knowledge regarding the processes affecting constituents of hair samples from ancient civilisations.

Results from the analyses indicate that the burial environment plays an important role in the postmortem variation in elemental content of the hair samples. Various elements demonstrate an ability to permeate through the hair matrix over time and hence cloud the true biogenic signal. Certain elements appear to have an affinity for the hair surface but an inability to permeate deeper. For example, iron on the surface of the KDT hair (Figure 2) and aluminium with the Pacatnamu mummies.

In addition, $\mathrm{NaCl}$ salt and what are believed to be aluminosilicates have been observed to accumulate on the surface of the Peruvian hairs. Various molecular fragments such as cysteic acid have been used to identify the extent and localisation of oxidation of the keratin proteins. Various features on the surface of the hair relating to contamination, accumulation and deterioration have been shown by ToF-SIMS analysis (Figure 3). Potential has also been found in the use of ToFSIMS for the measurement of isotopic values indicative of dietary habits. 

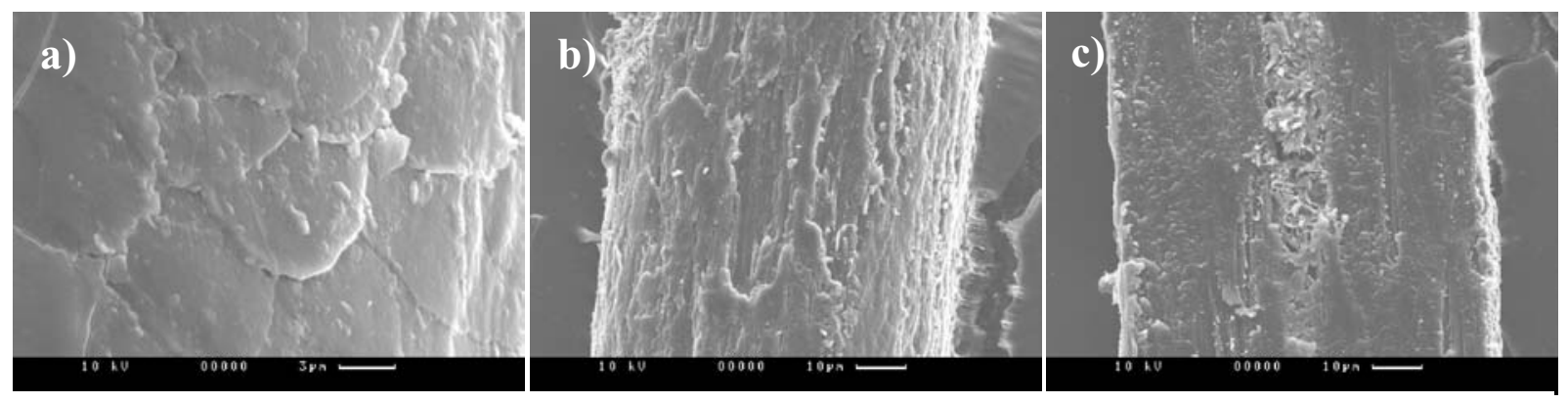

Figure 1: Hair proteins can remain very stable over long periods of time such as the 1000 year old (but dirty) sample in the left graphic. Other samples (b) can be the target of various degrading attacks. Longitudinal sectioning (c) and subsequent analysis however can discriminate between the surface contamination and internal concentrations.

\section{Longitudinal Internal and Surface Fe Concentrations in the KDT Hair}

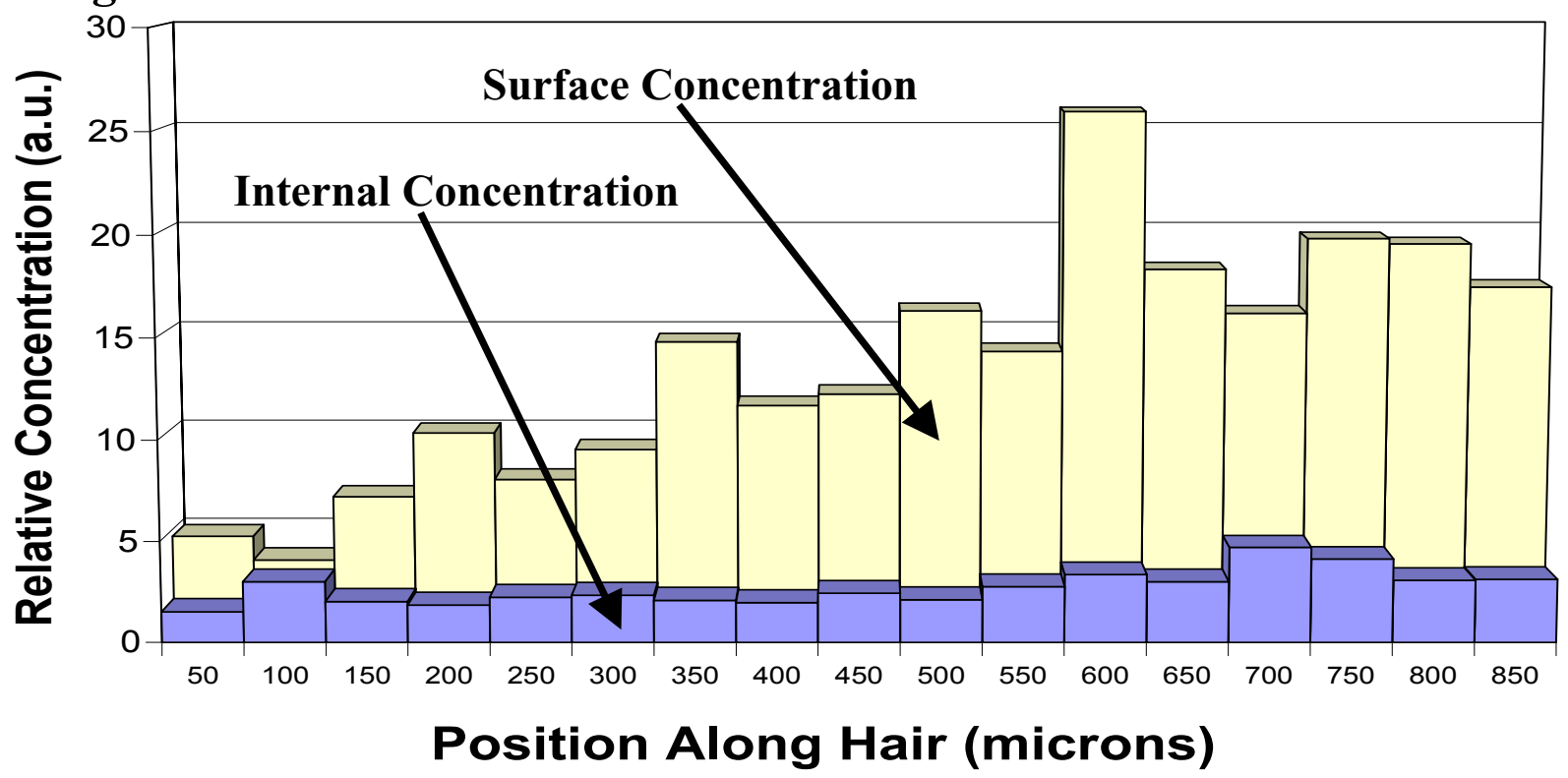

Figure 2: ToF-SIMS analysis demonstrates that Fe has accumulated on the surface of the KDT hair while the internal concentration remains relatively constant. A portion on the left of the graph was below the scalp level and hence little accumulation was restricted.
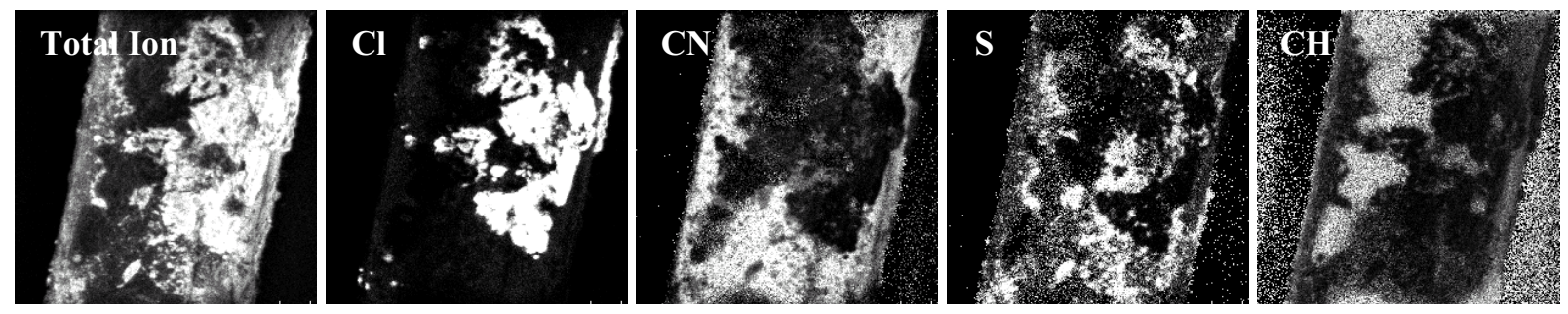

Figure 3: A selection of normalised images generated by ToF-SIMS showing discrete regions of $\mathrm{NaCl}$ salt, fragments of the protein backbone $(\mathrm{CN})$, areas of high oxidation $(\mathrm{S})$, and some contamination $(\mathrm{CH})$. 